\title{
Digital Accuracy in Comuputer Guided Surgery a Multicentre Prospective Study
}

\section{Fabrizia Luongo ( $\nabla$ fabrizialuongo@studioluongo.it )}

https://orcid.org/0000-0001-7998-613X

\section{Henriette Lerner}

Private practice Baden Baden Germany

\section{Camilla Gesso}

Private practice Albenga (SV)

\author{
Alessandro Motroni \\ 3Diemme srl Cantù (IT) \\ Alice Sormani \\ 3Diemme srl Cantù (Italy)

\section{Zamira Kalemaj} \\ private practice Milan
}

\section{Giovanni Polizzi}

Private practice BSC, Verona

\section{Giuseppe Luongo}

Department of Oral and Maxillofacial Surgery, Federico II University,Naples

\section{Research article}

Keywords: Computer Guided Surgery; Accuracy; Implantology; CBCT; IOS; Digital workflow

Posted Date: March 11th, 2020

DOI: https://doi.org/10.21203/rs.3.rs-16821/v1

License: (c) (i) This work is licensed under a Creative Commons Attribution 4.0 International License.

Read Full License 


\section{Abstract}

Background Digital technologies are becoming an irreplaceable assistance for a correct diagnosis and treatment planning of the dental therapy. Modern powerful software are able to match information coming from different sources (CBCT, IOS, lab and face scanners) creating a virtual patient where all the treatment options can be accurately tested. Guided surgery is based on the possibility to use these instruments to transfer the ideal plan directly into the operating theatre. However even in the hands of skilled operators there is always a difference between the position of the implant as determined on the virtual planning and the real position after the surgery. The aim of this multicenter prospective study is to test the advantages of the standardization of the digital workflow on the accuracy of the procedure. Methods 21 patients, for a total of 61 implants, have been treated in three different centers by operators with particular expertise in guided surgery. The accuracy of the procedure has been evaluated matching two files: the first from the pre-treatment virtual planning and the second from the optical impression taken immediately after the surgery. The digital workflow has been standardized using the same software for the planning, the same high quality manufacturing process, intraoral scanner and software for the overlapping procedure. Results All the implants showed an excellent primary stability. No intraoperative complications have been reported. The overlapping of the two files resulted in a mean angular deviation of $2.94^{\circ}$, a mean linear deviation at the platform of $0,73 \mathrm{~mm}$ and a mean vertical deviation at the apex of $0.01 \mathrm{~mm}$. Conclusions Almost the totality of the cases showed a better accuracy compared with the previous reported data of the literature. These results might be related with the standardization of the process so that following a precise protocol is mandatory for a safe use of this technique.

\section{Background}

The success of the implant therapy is closely related to the correct achievement of various parameters ${ }^{1-3}$. A correct position of the implant and the best emergence profile are just two of the most important aspects to reach an aesthetic and functional implant-supported restoration ${ }^{4-6}$.

During the years, many techniques have been proposed for a precise implant position analysis ${ }^{7}$.

The perfect positioning of an implant and the impact of its emergence in relation with an optimal prosthetic result can be evaluated using the modern software for treatment planning ${ }^{8,9}$. These powerful software packages are able to match the anatomical information coming from 3D radiological devices (СBCT, СТ...) with the wax up showing the ideal final outcome.

In this way, the clinicians can work on a clear 3D view of the ideal implant position in terms of depth, angulation and diameter ${ }^{10}$

With the diffusion of the virtual planning, two different options became available:

- static approach is performed using a surgical template which is manufactured directly from the virtual planning; 
- dynamic approach allows the clinician to follow a surgical navigator assisted free hand surgery while checking onscreen the planning prepared on the virtual plan ${ }^{11}$.

In expert hand, these solutions do not show significant differences; anyhow, the general trend is in support of the stent technique. ${ }^{12}$

The static approach has the main advantage that it does not need using additional expansive equipment; while it has the drawback of not having a free view of the surgical field (most times this technique is flapless). Moreover, no changes on the implant positioning can be done during the surgery, which instead is possible with the navigation.

High accuracy of the stent technique can be gained by following a standard workflow: each step of the protocol must be completed carefully, in order to reduce extrinsic and intrinsic errors. ${ }^{13}$ All the errors collected during the workflow procedure can lead to a severe difference of the position of the implants at the end of the surgery. ${ }^{14}$

Combining the anatomical information from the CT/ CBCT with the ones from the wax up, it is possible to create an ideal virtual planning. A total digital workflow allows the manufacture of a surgical stent designed directly from the 3D implant planning. ${ }^{15,16}$.

Two different procedures can be used in order to combine this essential information for a correct diagnosis and treatment planning:

1. In the Double-scan technique, data retrieved from a $\mathrm{CBCT}$ of the patient wearing a radiological stent are matched with the ones from a second CBCT of the stent alone. ${ }^{17}$

A specific software matches the two files, so it is possible to obtain a 3D visualization of bone anatomy and ideal prosthetic outcome.

Through this complete view, clinician can prepare a virtual planning for the insertion of the implants and afterwards send the project to a manufacturing center for the surgical guide manufacturing

2. The "Fusion Technique" is based on the match between patient data from a CBCT and the ones from an intraoral scan of the patient dentition ${ }^{18}$,

As an alternative the overlapping can be done between the patient's CBCT and the scan of the patient's gums cast model realized with an analogical impression. ${ }^{19}$

These files are matched together into a specific software in the same way of the double scan technique. A virtual or an analogic wax up is then added and a planning is prepared for the final manufacturing of the surgical stent. 
In both ways, the stent can then be manipulated through a reverse flow chart in order to create a working model. It is used for the analogic creation of a temporary prosthesis to be applied immediately after surgery ${ }^{20}$.

The Computer Guided Surgery can be defined as a procedure that uses computer-aided design and computer-aided manufacturing (CAD/CAM) technology to fabricate a surgical stent that guides the surgeon during the implant insertion procedure.

It has been demonstrated that accuracy of a guided implants' placement is significantly higher, if compared with non-guided surgical procedure ${ }^{21}$.

Anyhow, being a blind procedure, it is extremely important to be as accurate as possible to reduce the difference between the virtual and the real position of the implant.

In fact, some linear and angular deviations between the planned and placed implants can be expected even when performed by experienced clinicians ${ }^{22}$.

Many studies reported different levels of discrepancy between the virtual and the real position of the implants in guided surgery, which depend on many variables and not only on the clinician's experience. The most important factors involved in the arising of errors are 3D radiological examination quality, correct virtual planning, stent manufacturing procedures, transfer and fixation of the guide into the surgical field. ${ }^{23}$

Most of published studies analyze the deviation between the planned implants and the placed ones superimposing the virtual planning with a second CBCT at the end of the surgery ${ }^{24}$.

This solution obliges the patient to an unnecessary second $x$-ray exposure which has been firmly criticized $^{25}$.

Son K. and co-workers proposed a different and more ethical solution: they matched two STL files retrieved by the virtual plans and the intraoral scan of the placed implant. ${ }^{26}$

The aim of this multicenter prospective study is to evaluate the accuracy of the Guided Implant Surgery using this less invasive procedure.

\section{Methods}

Between June 2018 and October 2019, a total of 21 patients were enrolled in the study. All the patients underwent treatment using stent computer guided systems.

The surgeries have been done in three different centers in Europe.

All the patients signed an informed consent. 
Both total and partial edentulous cases were treated. One surgeon at each center performed the surgery.

The inclusion criteria were:

- Male or female > 18 years old;

- Correct bone quantity at the implant site to permit the insertion of at least 1 implant with a $3.3 \mathrm{~mm}$ minimum diameter of and at least $7 \mathrm{~mm}$ minimum length;

- Enough patient mouth opening for using the specific guided surgery tools;

- Correct soft and hard tissues volume;

- Deemed by the investigator to be suitable for guided implant surgery procedure.

All patients showing medical conditions not compatible with surgical implant treatment have been excluded from the study.

Smokers of more than 10 cigarettes per day were also not included.

All the patients underwent a CBCT scan for the 3D acquisition of bone anatomy. The file format for CBCT axial images is standard DICOM.

The double scan technique for full edentulous cases and fusion technique for single or partial edentulous rehabilitations were followed.

For all the patients requiring fusion procedure an analogic or a virtual wax up were acquired. Analogic information was then scanned with a laboratory table-top scan in order to produce a 3D STL file.

Both wax-up STL and CBCT DICOM files were later imported and matched together in a 3D-planning software (Real Guide 5.0 - 3DIEMME srl, via Risorgimento, 9 - 22063 Cantù (CO) - ITALY).

All the centers used the same software in the planning phase, while different companies (Megagen - CTech - i-Res - JD Implants) provided implants and instruments for surgery.

Dental implants' ideal positions were then planned by the surgeons and prosthodontics.

The final virtual planning was sent to the company (3DIEMME srl, via Risorgimento, 9 - 22063 Cantù (CO) - ITALY)) for the surgical stent production. In order to uniform the analysis, the same lab and printer have been used (Stratasys Eden260VS Dental Advantage, Eden Prairie, Minnesota; United States)

The clinician independently arranged the production of the provisional restoration for an immediate loading procedure, if planned.

After the surgical step, an indirect method for measuring the accuracy was performed.

Intra-oral scans of the mouth of each patient were performed: acquisitions included gums anatomy and standard dedicated scan-bodies too, which were positioned on top of every implant. 
Two centers used the same intra-oral scanner (Carestream 3600 - Carestream Dental LLC 3625 Cumberland Blvd. Ste. 700 Atlanta, GA 30339) while the third center used a different one (i500 Medit MEDIT corp. 23 Goryeodae-ro 22 gil, Seongbuk-gu, Seoul, Korea).

For each patient the acquired information was:

1. Gums anatomy before surgery STL file;

2. Gums anatomy with scan-body information after surgery STL file.

In order to make a comparison between the planned implants' position and the real situation in the mouth of the patient, the after surgery STL file was then matched to the before surgery one.

\section{Superimposition}

Superimposition or matching is a procedure that allows to overlap the virtual position of the implant as determined in the virtual planning software with the real situation after surgery.

For each patient, the process steps are the following:

1. Get the implants' position and geometry together with theirs scan-abutment from the virtual planning. The information on implants and scan-abutments were available in the software implants library and were provided by the manufacturers themselves;

2. Get the patient's anatomy STL files from the virtual planning (it is in the correct position with respect to the CBCT bone information and of course implants position);

3. Get the intra-oral scan of the patient's mouth also comprising the implant scan-abutments (aftersurgery anatomy), as explained in the previous paragraph;

4. Match the after-surgery anatomy file with the before-surgery one, by selection of surface areas not affected by the surgery itself: the two anatomy files (before and after surgery) were thus placed in the same position;

5. Thanks to the scan-abutment placing in both the before-surgery and after-surgery environments, we were able to deduce the after-surgery implants' positions;

6. Thanks to the scan-abutment present in both before-surgery and after-surgery environments, we were able to deduce the after-surgery implants' positions;

7. From the before-surgery and after-surgery implants' position, we were able to evaluate deviations.

In order to get useful data from each patient, it is important to arrange before-surgery and after-surgery 3D objects in a same 3D reference system: after-surgery information was moved into before-surgery reference system.

In order to do that, superimposition of matching areas from the before and after surgery optical scan was requires: these areas needed to be as wide as possible and not affected by the surgery, for example teeth in partial edentulous patients, or hard palate in complete edentulous maxilla. 
GeoMagic Wrap 12 software (3D Systems, Rock Hill, South Carolina, USA) function "Best-fit Alignment" was used in this study. This function can set the most precise position of the after surgery oral scan with respect of the before surgery one, the reference object.

The next step was to deduce the after surgery implants position from the scan-abutment geometry: in fact, knowing their shape and their height with respect to the fixture platform (it is available in the planning software implants' library), we were able to get the implants' position in the after surgery oral scan.

We used GeoMagic Wrap 12 software function Best fit Alignment again, easily applied to the asymmetric scan-abutment geometry.

\section{Data Analysis}

In order to analyze accuracy and precision of RealGUIDE protocol, we took into consideration the following parameters for every implant, with respect with the virtually planned position:

1. linear deviations at the implant platform;

2. linear deviations at the implant apex;

3. implant axes angular deviation;

4. vertical deviation at the implant platform;

5. vertical deviation at the implant apex.

We simplified implants geometries turning them into cylinders, so it was possible to determine linear deviations of the after surgery situation (with respect to the before surgery implant position) as distances between centres of bases and apexes: (see Formula 1 in the Supplementary Files)

For every couple of implants, we measured the distance between centers at apex and platform (Figure 3), which represent the deviations of the after-surgery implants (green) compared with the planned ones (yellow).

The accuracy of a guided implant system is higher the more the deviations are small. The precision is higher the more the set of deviation values is close to the mean.

The mean is calculated as follows: (see Formula 2 in the Supplementary Files)

Where $\mathrm{n}_{1}, \mathrm{n}_{2}, \ldots, \mathrm{n}_{\mathrm{N}}$ are the values of a dataset;

$\mathrm{N}$ is the number (integer) of data available.

One more parameter evaluated in this study is the center of mass of the virtual guide compared with the one of the real guide into each patient's mouth. This measure is important to detect guide's positioning errors in the surgical step. 


\section{Results}

Twenty-one patients between 18 and 75 years old were treated receiving an overall of 61 implants.

Three cases were total edentulous ,16 partial edentulous, while only 2 were single missing tooth.

Center 1 treated 5 patients with 20 implants, center 2 treated 8 patients with 25 implants, and center 3 treated 8 patients with 16 implants.

Each patient received 1 to 6 implants according to the type of edentulism. Thirty-six implants were placed on the maxilla, of which 15 in the anterior region and the rest in the posterior one. Whereas, all 25 mandibular implants were positioned in the posterior area.

In all the total edentulism cases (three patients) the surgical guide was mucosa supported; in all other cases the surgical guide was teeth supported.

Descriptive summary statistics for all linear and angular deviations are reported in Table 1. The most important mean deviation was observed at the apex level (mean 1.06, SD 0.53) not due to a vertical deviation at this level (mean 0.01, SD 0.78).

Table 1: Descriptive summary statistics for all measured deviations

\begin{tabular}{|l|c|c|c|c|c|}
\hline & Mean & $\begin{array}{c}\text { Standard } \\
\text { Deviation }\end{array}$ & Median & Range & $\begin{array}{c}\text { 95\% Confidence } \\
\text { Interval }\end{array}$ \\
\hline $\begin{array}{l}\text { Linear platform } \\
(\mathrm{mm})\end{array}$ & 0.73 & 0.3 & 0.72 & $\begin{array}{c}0.16 \text { to } \\
1.63\end{array}$ & 0.34 to 1.36 \\
\hline Linear apex (mm) & 1.06 & 0.53 & 0.89 & $\begin{array}{c}0.25 \text { to } \\
3.27\end{array}$ & 0.35 to 1.85 \\
\hline $\begin{array}{l}\text { Vertical platform } \\
\text { (mm) }\end{array}$ & 0.29 & 0.44 & 0.17 & $\begin{array}{c}-1.26 \text { to } \\
0.93\end{array}$ & -0.59 to 0.75 \\
\hline Vertical apex (mm) & 0.01 & 0.78 & 0.28 & -3.15 to & -0.89 to 1.24 \\
\hline Angular (degrees) & 2.94 & 1.84 & 2.38 & 0.68 to & 1.04 to 5.5 \\
\hline
\end{tabular}

Multilevel regressions, accounting for center and patient clustering indicated no relevant difference between maxillary and mandibular arch and type of support for any of the measured outcomes (Table 2). 
Implants positioned posteriorly seemed to exhibit higher deviation at implant platform (Coeff. 0.18, $\mathrm{P}=0.036$ ) and higher angular deviation (Coeff. $1.35, \mathrm{P}=0.012$ ) compared to implants positioned anteriorly.

Table 2: Results of multilevel regression models for each outcome and potential predictor variable

\begin{tabular}{|l|l|l|l|l|l|l|}
\hline & \multicolumn{2}{|l|}{$\begin{array}{l}\text { Mandibular arch vs. } \\
\text { maxillary arch }\end{array}$} & \multicolumn{2}{l}{$\begin{array}{l}\text { Tooth support vs. } \\
\text { mucosal support }\end{array}$} & $\begin{array}{l}\text { Posterior position vs. } \\
\text { anterior position }\end{array}$ \\
\hline Deviations & Coefficient & $P$-value & Coefficient & P-value & Coefficient & $P$-value \\
\hline $\begin{array}{l}\text { Linear } \\
\text { platform (mm) }\end{array}$ & 0.12 & 0.13 & 0.001 & 0.99 & 0.18 & $0.036^{*}$ \\
\hline $\begin{array}{l}\text { Linear apex } \\
\text { (mm) }\end{array}$ & 0.02 & 0.43 & 0.04 & 0.81 & 0.08 & 0.35 \\
\hline $\begin{array}{l}\text { Vertical } \\
\text { platform (mm) }\end{array}$ & 0.01 & 0.93 & 0.15 & 0.28 & 0.05 & 0.69 \\
\hline $\begin{array}{l}\text { Vertical apex } \\
(\mathrm{mm})\end{array}$ & 0.01 & 0.67 & 0.03 & 0.17 & 0.1 & 0.66 \\
\hline $\begin{array}{l}\text { Angular } \\
\text { (degrees) }\end{array}$ & 0.54 & 0.31 & -0.34 & 0.58 & 1.35 & $0.012^{*}$ \\
\hline
\end{tabular}

*Statistically significant

Mean barycenter deviation was $0.67 \mathrm{~mm}$ (SD 0.47 ), ranging from 0.14 to $2.56,95 \% \mathrm{Cl} 0.24$ to 1.44 .

Correlation analysis between different deviations are presented in Table 3. The highest correlation coefficients were found between the two linear deviations (Correlation coeff. 0.68) and the two apical deviations (Correlation coeff. 0.69) respectively. A certain positive correlation was also observed between angulation and linear apex deviation (Correlation coeff 0.67 ).

Table 3: Pairwise correlation between different deviations 


\begin{tabular}{|c|c|c|c|c|c|c|}
\hline & $\begin{array}{l}\text { Linear } \\
\text { platform }\end{array}$ & $\begin{array}{l}\text { Linear } \\
\text { apex }\end{array}$ & $\begin{array}{l}\text { Vertical } \\
\text { platform }\end{array}$ & $\begin{array}{l}\text { Vertical } \\
\text { apex }\end{array}$ & Angulation & Barycenter \\
\hline $\begin{array}{c}\text { Linear } \\
\text { platform }\end{array}$ & 1 & & & & & \\
\hline Linear apex & 0.68 & 1 & & & & \\
\hline $\begin{array}{l}\text { Vertical } \\
\text { platform }\end{array}$ & 0.19 & 0.04 & 1 & & & \\
\hline Vertical apex & -0.09 & -0.09 & 0.69 & 1 & & \\
\hline Angulation & 0.19 & 0.67 & -0.20 & -0.14 & 1 & \\
\hline Barycenter & 0.39 & 0.25 & 0.14 & 0.10 & -0.09 & 1 \\
\hline
\end{tabular}

\section{Discussion}

It is proved that the use of software for planning implant position can give to the clinician a more predictable outcome of the prosthetic result ${ }^{27}$.

Computer guided surgery technique has many benefits:

- Possibility of using a flapless approach, a less invasive procedure for the patient;

- One step option;

- Reduced surgical procedure times;

- More precise and accurate osteotomy, leading to reduced pain and swelling.

Several authors demonstrated that the computer guided surgery is a predictable technique ${ }^{28,29}$.

Chmielewski and co-workers ${ }^{30}$ analyzed the surgical approach in full-arch rehabilitation with dental implants and immediate loading using digital workflow, and concluded that a full implant-retained fixed prosthetics with the help of digital workflow becomes a predictable and fast solution for both dentists and patients. Digital workflow successfully enabled the immediate full-arch rehabilitation with a predictable outcome by different surgeons in multiple centers.

Accuracy and precision of the computer guided systems is also well demonstrated.

Van D'Haese and co-workers in 2102, studying the accuracy of guided implant surgery system using mucosa supported stents, have shown a mean angular deviation of $2.6^{\circ}$ and a mean cervical deviation and apical deviation respectively of $0.91 \mathrm{~mm}$ e $1.13 \mathrm{~mm}^{31}$

However even in expert hands there is always a discrepancy between the virtual planned implant position and the real one ${ }^{32}$. 
In fact, different potential sources of errors during the examination, the planning, the manufacturing and the execution phase can be collected.

For example, during the 3D x-ray examination, errors can be made due to patient movements, quality of the fitting of the scan prosthesis or presence of metal artefacts.

Peterson and co-workers reported that $40 \%$ of the patients move during the examination leading to artefacts. $^{33}$

The fit of the scan prosthesis with the patient soft tissues needs to be perfectly check before the scan execution.

No air between the scan prosthesis and the soft tissues must be present in the scout view before going ahead with the examination.

The use of a radiological index to be worn by the patient during the examination can reduce significantly the misfit of the scan prothesis. ${ }^{34}$

Metal artefacts can also influence the image quality making more difficult the identification of the outline of the alveolar bone and anatomical boundaries ${ }^{35}$

Errors can also occur during the elaboration of the virtual planning especially in the most complicated cases like total edentulism. In these cases, clinician needs to choose a maximum number of asymmetric registered markers on the radiological stent so to grant an easier ad more reliable matching procedure.

A manual correction of the matching of fiducial markers, in particular in case of automatic superimposition procedure, is often needed. ${ }^{36}$

The production process of the surgical stent is also a critical point. This feature should be left to skilled manufacturers who can rely on advanced technologies. ${ }^{37-39}$

Since 1993 Kruth $^{40}$ observed that there is always a deviation in the production process of a stereolithographic guide with a mean value of $0.3 \mathrm{~mm}$.

Dreiseidler and co-workers ${ }^{41}$ came to the same conclusions showing an overall deviation of about 0.5 $\mathrm{mm}$ in the laboratory production process.

A wrong positioning and stabilization of the surgical template before surgery can also be a source of errors.

Especially in case of total edentulism this step could be really challenging. For this reason, in order to reduce misfits using a surgical index and fixation pins is a mandatory step. ${ }^{42}$

The removal of the stent during the surgical procedure can be also a reason of losing precision. ${ }^{43}$ 
That's why it is suggested not to remove the stent during the drilling and implant placement procedure.

Guidance of the implant during the insertion is system specific, we should prefer systems with physical stop then visual ones.

\section{Methods to evaluate difference between virtual implant position and real one:}

Discrepancies between the position of the virtual implants and the real one detection can be detected thought different techniques.

1. Direct technique approach consists of the execution of a second CBCT exam of the patient, right after the surgical procedure of implant placement, in order to radiographically find the implants positions in space.

In this case, the titanium material which the implant is made of, is usually cause of metal artefacts and background noise in the CBCT DICOM images, thus leading to a much more complex superimposition.

As a result, confirmation of accurate implant positioning is possible only by estimation. The artefacts coming from the material of the implant give an augmentation of the implant margins that may cause errors in the results.

In order to reduce errors in the matching of the implants, the use of at least three different reference points is mandatory. In case of partial edentulism we can usually detect three natural residual teeth as reference points. Another possibility is using three radiopaque markers during the before and after surgery CBCT exams.

In case of total edentulism, the anatomic structures are not very well identified on the $\mathrm{CT}$, so radiopaque markers are needed as accurate reference points.

The accuracy of the CT images is also strongly dependent on the type of device used for the x-ray exam, so using the same CBCT before and after the surgical procedure is necessary.

Some ethic problems related to the direct evaluation method must also be taken into consideration.

CBCT could be justified for presurgical diagnosis, preoperative planning and preoperative transfer for oral implant rehabilitation

The use of CBCT imaging following insertion of dental implants should be restricted to specific postoperative complications (such as iatrogenic neurovascular trauma), required implant retrieval and followup of complex surgical procedures.

2. Indirect technique can be followed by connecting the scan bodies to the implant and scan them with the use of an IOS device. Obtaining an optical impression, the patient doesn't need to take another CBCT after the surgery reducing greatly the amount of radiation exposure. 
An impression is taken right after the surgery or when the rehabilitated area is completely healed.

This technique can indirectly register the real position of the implants by matching the scan body with the fixture: the correct virtual position between scan-body and implant is achieved using information provided by manufacturers themselves (height of Implant scanbody connection) and already included in the 3D planning software implants library.

The discrepancy between the real and the planned implant position is detected overlapping two pieces of the same 3dimensional area on a single plane using 3 or more different reference points that are not in a straight line.

In partial edentulous cases we can usually identify at least three residual teeth as reference points. In total edentulism cases we need special reference points such as the hard palatal area.

In this study the indirect method has been used, while most of the published articles applied a radiological approach (direct technique) for the evaluation of the accuracy. ${ }^{44}$

Marliere and co-authors ${ }^{45}$ in a recent systematic review evaluate the accuracy of computer-guided surgery in 7 studies. All these studies included only prospective or retrospective clinical trials with a minimum of 5 full edentulous patients in maxilla or mandible excluding studies based on cadavers or models controlled or randomized. The average of the angular deviation ranged between $1.85^{\circ}$ to $8.4^{\circ}$.

Petterson and co-workers ${ }^{46}$ analyzed mucosa supported cases and found out a mean value of linear cervical deviation of $0.80 \mathrm{~mm}$.

Strubinger and co-authors ${ }^{47}$ used a screw-fixed bone-supported guide in an open flap surgical approach

They detected a lower value of cervical linear deviation of $0.71 \mathrm{~mm}$, which might be related to the bone supported guides that are more stable than mucosa supported ones.

Cassetta et al. ${ }^{48}[20]$ evaluated the precision of muco-supported surgical guides with and without fixation screws in the edentulous edges of the maxilla and mandible. In a paired comparison between the means of the deviations (with or without fixation), they showed that the fixed guides resulted in better precision of implant placement, which was statistically significant for angular deviation (angular: with-4.09 , without $-5.62^{\circ}$ ). They concluded that fixation of the surgical guides allows greater stability, reducing errors between the planned and the executed treatment.

Recently some authors reported good accuracy values using an IO scanner to determine the real position of the implants after the surgery. ${ }^{49-51}$

Skjerven and coworkers ${ }^{52}$ compared these two techniques concluding that there are no differences in terms of accuracy measurement. 
Our study was then performed using the optical impression of the implant position as a reference.

We found mean angular deviation of $2.94^{\circ}$ which is in line with the best results reported in the current literature.

We have to consider that two cases showed very high values (8.12 and 10.07) while the mean results were in an average of less than 2 degrees.

The $95 \%$ confidence interval was between $1.04^{\circ}$ to $5.5^{\circ}$.

The mean deviation at the platform level was $0.73 \mathrm{~mm}$ with a range of 0.16 to 1.63 while the mean vertical deviation at the apex was $0,01 \mathrm{~mm}$ with a range between -3.15 to $1.9 \mathrm{~mm}$. The $95 \%$ confident interval was between -0.89 to $1.24 \mathrm{~mm}$.

These excellent results can be related to the standardization of the process using the same software for all the centers and same manufacturing Company (3DIEMME srl, via Risorgimento, 9 - 22063 Cantù (CO) - ITALY).

No significant difference between type of support (tooth or mucosa) has been found (Table 2).

On the other hand, relevant differences were found between implants positioned in the anterior or posterior regions. Higher linear implant platform and angular deviation was showed in the posterior area (respectively coefficient 0.18 and P-value 0.036 and coefficient 1.35 and P-value 0.012 ) compared to the anterior area.

This might be related to the presence of a low-density bone (posterior maxilla region) which seems to influence the entity of the deviation.

Anyhow there is no statistical significantly difference comparing the implants placed in the maxilla and in the mandibula with a mean angular deviation coefficient of 0.54 and a p-value of 0.31 .

D'Haese et al. ${ }^{31}$ highlighted that if deviation in angulation occurred, by mathematical reasoning, there should be greater deviation at the apex of the implant

In line with D'Haese consideration, a positive correlation was observed between angulation and linear apex deviation with a correlation coefficient of 0.67 .

\section{Conclusions}

According to the recent literature ${ }^{52}$ this study shows that the valuation of the accuracy by using an 10 scanner overlapped with the virtual plan is a practical method for analysis.

Better results presented in this study with respect to previous published data could be related to the strict protocol, which included a single software for treatments' planning and a high-quality manufacturing 
process.

Anyhow, further studies are needed to find out strategies to improve the accuracy and the overall outcome of the guided surgery procedures.

\section{Declarations}

\section{Ethics approval and consent to participate}

No formal ethics approval was required in this particular case as all the treatments has followed the standard rules for the routine therapies in daily practice.

\section{Consent for publication}

All the operators gave their agreements for the data publication.

\section{Availability of data and material}

The datasets used and/or analysed during the current study are available from the corresponding author on reasonable request.

\section{Competing interests}

The authors declare that they have no competing interests.

\section{Funding}

This study was neither financially and not financially supported.

\section{Authors' contributions}

FL have designed the study and provided cases included in the study, $\mathrm{HL}$ provided cases included in the study, CG provided cases included in the study, AM participated to the data analysis, AS participated to the data analysis, KZ performed the statistical analysis, GP provided cases included in the study, GL participated to the design of the study and provided cases included in the study.

\section{Acknowledgements}

Not applicable.

\section{References}

1. Cooper LF, Reside GJ, Raes F, Garriga JS, Tarrida LG, Wiltfang J, Kern M, De Bruyn H. “Immediate provisionalization of dental implants placed in healed alveolar ridges and extraction sockets: a 5- 
year prospective evaluation." Int J Oral Maxillofac Implants. 2014 May-Jun;29(3):709-17.

2. De Bruyn H, Raes S, Ostman PO, Cosyn J. "Immediate loading in partially and completely edentulous jaws: a review of the literature with clinical guidelines." Periodontol 2000. 2014 Oct;66(1):153-87

3. Vázquez Álvarez R, Pérez Sayáns M, Gayoso Diz P, García García A. “Factors affecting peri-implant bone loss: a post-five-year retrospective study." Clin Oral Implants Res. 2015 Sep;26(9):1006-14. Epub 2014 Jun 30.

4. Akin R. J Oral Maxillofac Surg. "A New Concept in Maintaining the Emergence Profile in Immediate Posterior Implant Placement: The Anatomic Harmony Abutment." 2016 Dec;74(12):2385-2392. Epub 2016 Jul 1.

5. Steigmann M, Monje A, Chan HL, Wang HL. "Emergence profile design based on implant position in the esthetic zone." Int J Periodontics Restorative Dent. 2014 Jul-Aug;34(4):559-63.

6. Becker W, Doerr J, Becker BE. "A novel method for creating an optimal emergence profile adjacent to dental implants." J Esthet Restor Dent. 2012 Dec;24(6):395-400. Epub 2012 Jul 2.

7. Schubert O, Schweiger J, Stimmelmayr M, Nold E, Güth JF. Digital implant planning and guided implant surgery - workflow and reliability. Br Dent J. 2019 Jan 25;226(2):101-108.

8. Joda T, Wittneben JG, Brägger U, "Digital implant impressions with the "Individualized Scanbody Technique" for emergence profile support." Clin Oral Implants Res. 2014 Mar;25(3):395-7. Epub 2013 Jan 4.

9. Widmann G, Widmann R, Widmann E, Jaschke W, Bale R. "Use of a surgical navigation system for CT-guided template production." Int J Oral Maxillofac Implants. 2007 Jan-Feb;22(1):72-8.

10. Jung, R.E.; Schneider, D.; Ganeles, J.; Wismeijer, D.; Zwahlen, M.; Hämmerle, C.H.; Tahmaseb, A. Computer technology applications in surgical implant dentistry: A systematic review. Int. J. Oral Maxillofac. Implants 2009, 24, 92-109.

11. Pellegrino G, Taraschi V, Andrea Z, Ferri A, Marchetti C. Dynamic navigation: a prospective clinical trial to evaluate the accuracy of implant placement. Int J Comput Dent. 2019;22(2):139-147

12. Vercruyssen, M.; Fortin, T.; Widmann, G.; Jacobs, R.; Quirynen, M. Different techniques of static/dynamic guided implant surgery: Modalities and indications. Periodontology 2014, 66, 214227.

13. Tatakis DN, Chien HH, Parashis AO. Guided implant surgery risks and their prevention. Periodontol 2000. 2019 Oct;81(1):194-208. 
14. Vieira, D.M.; Sotto-Maior, B.S.; Barros, C.A.; Reis, E.S.; Francischone, C.E. Clinical accuracy of flapless computer-guided surgery for implant placement in edentulous arches. Int. J. Oral Maxillofac. Implants 2013, 28, 1347-1351

15. Duda M, Neugebauer J, Stachulla G, Ormianer Z, "A novel method of preparation of surgical template for guided implant placement: a case report. Implant Dent. 2011 Apr;20(2):118-24.

16. Widmann G, Berggren JP, Fischer B, Pichler-Dennhardt AR, Schullian P, Bale R, Puelacher W. "Accuracy of Image-Fusion Stereolithographic Guides: Mapping CT Data with Three-Dimensional Optical Surface Scanning." Clin Implant Dent Relat Res. 2015 Oct;17 Suppl 2:e736-44 Epub 2015 Mar 17.

17. Lanis A, Llorens P, Álvarez Del Canto O. "Selecting the appropriate digital planning pathway for computer-guided implant surgery." Int J Comput Dent. 2017;20(1):75-85.

18. Oh JH, An X, Jeong SM, Choi BH. "Digital Workflow for Computer-Guided Implant Surgery in Edentulous Patients: A Case Report." J Oral Maxillofac Surg. 2017 Dec;75(12):2541-2549. Epub 2017 Aug 12.

19. Yilmaz B. Incorporating digital scans of diagnostic casts into computed tomography for virtual implant treatment planning. J Prosthet Dent. 2015 Aug;114(2):178-81.Epub 2015 Apr 29.

20. Park SJ, Leesungbok R, Cui T, Lee SW, Ahn SJ. "Reliability of a CAD/CAM Surgical Guide for Implant Placement: An In Vitro Comparison of Surgeons' Experience Levels and Implant Sites." Int J Prosthodont. 2017 Jul/Aug;30(4):367-169

21. Nickenig HJ, Wichmann M, Hamel J, Schlegel KA, Eitner S. "Evaluation of the difference in accuracy between implant placement by virtual planning data and surgical guide templates versus the conventional free-hand method - a combined in vivo - in vitro technique using cone-beam CT (Part II)." J Craniomaxillofac Surg. 2010 Oct;38(7):488-93. Epub 2009 Nov 24.

22. Hinckfuss S, Conrad HJ, Lin L, Lunos S, Seong WJ. Effect of surgical guide design and surgeon's experience on the accuracy of implant placement. J Oral Implantol. 2012 Aug;38(4):311-23.

23. Bover-Ramos F, Viña-Almunia J, Cervera-Ballester J, Peñarrocha-Diago M, García-Mira B.Accuracy of Implant Placement with Computer-Guided Surgery: A Systematic Review and Meta-Analysis Comparing Cadaver, Clinical, and In Vitro Studies. Int J Oral Maxillofac Implants. 2018 January/February;33(1):101-115. doi: 10.11607/jomi.5556. Epub 2017 Jun 20. Review.

24. Valente F, Schiroli G, Sbrenna A. Accuracy of computer-aided oral implant surgery: A clinical and radiographic study. Int J Oral Maxillofac Implants. 2009;24:234-42.

25. Vercruyssen M, Laleman I, Jacobs R, Quirynen M. “Computer-supported implant planning and guided surgery: a narrative review.” Clin Oral Implants Res. 2015 Sep;26 Suppl 11:69-76. 
26. Son K, Huang MY, Lee KB. A method to evaluate the accuracy of dental implant placement without postoperative radiography after computer-guided implant surgery: A dental technique. J Prosthet Dent. 2019 Aug 2. pii: S0022-3913(19)30296-3.[Epub ahead of print]

27. D'haese J, Ackhurst J, Wismeijer D, De Bruyn H, Tahmaseb A. Periodontol 2000. 2017 Feb;73(1):121133. doi: $10.1111 /$ prd.12175.Current state of the art of computer-guided implant surgery..

28. Van Assche N, van Steenberghe D, Quirynen M, Jacobs R. Accuracy assessment of computerassisted flapless implant placement in partial edentulism. J Clin2010 Apr;37(4):398-403.

29. Seo C, Juodzbalys G Accuracy of Guided Surgery via Stereolithographic Mucosa-Supported Surgical Guide in Implant Surgery for Edentulous Patient: a Systematic Review. J Oral Maxillofac Res. 2018 Mar 31;9(1):e1.

30. Chmielewski K, Ryncarz W, Yüksel O, Goncalves P, Baek KW, Cok S, Dard M. Image analysis of immediate full-arch prosthetic rehabilitations guided by a digital workflow: assessment of the discrepancy between planning and execution. Int J Implant Dent. 2019 Jul 15;5(1):26.

31. D'haese J1, Van De Velde T, Elaut L, De Bruyn H A prospective study on the accuracy of mucosally supported stereolithographic surgical guides in fully edentulous maxillae. Clin Implant Dent Relat Res. 2012 Apr;14(2):293-303

32. Testori T, Robiony M, Parenti A, Luongo G, Rosenfeld AL, Ganz SD, Mandelaris GA, Del Fabbro M. Evaluation of accuracy and precision of a new guided surgery system: a multicenter clinical study. Int J Periodontics Restorative Dent. 2014;34 Suppl 3:s59-69.

33. Drago C, del Castillo R, Peterson T. J Prosthodont. Immediate occlusal loading in edentulous jaws, CT-guided surgery and fixed provisional prosthesis: a maxillary arch clinical report. 2011 Apr;20(3):209-17.

34. De Santis D, Graziani P, Castellani R, Zanotti G, Gelpi F, Marconcini S, Bertossi D, Nocini PF. A New Radiologic Protocol and a New Occlusal Radiographic Index for Computer-Guided Implant Surgery.De Santis D et al. J Craniofac Surg. 2016 Jul;27(5):e506-10.

35. Behneke, A.; Burwinkel, M.; Behneke, N. Factors influencing transfer accuracy of cone beam CTderived template-based implant placement. Clin. Oral Implants Res. 2012, 23, 416-423.

36. Rußig LL, Schulze RK. Effects of minute misregistrations of prefabricated markers for image-guided dental implant surgery: an analytical evaluation. Clin Oral Implants Res. 2013 Dec;24(12):1339-46.

37. Matta RE, Bergauer B, Adler W, Wichmann M, Nickenig HJ. The impact of the fabrication method on the three-dimensional accuracy of an implant surgery template. J Craniomaxillofac Surg. 2017 Jun;45(6):804-808. 
38. Stumpel LJ. Deformation of stereolithographically produced surgical guides: an observational case series report. Clin Implant Dent Relat Res. 2012 Jun;14(3):442-53.

39. Jiang Y, Zhang ZH, Liu HH, Zhou HT, Rui X, Zhang YY. Hua Xi Kou Qiang Yi Xue Za Zhi. Comparative study of 3D printing implant guide in different implant surgeries in anterior tooth defect area]. 2019 Aug 1;37(4):403-407.

40. Ruth, V.; Kolditz, D.; Steiding, C.; Kalender, W.A. Metal Artifact Reduction in X-ray Computed Tomography Using Computer-Aided Design Data of Implants as Prior Information. Invest. Radiol. 2017, 52, 349-359.

41. Dreiseidler T, Neugebauer J, Ritter L, Lingohr T, Rothamel D, Mischkowski RA, Zöller JE. Accuracy of a newly developed integrated system for dental implant planning. Clin Oral Implants Res. 2009 Nov;20(11):1191-9)

42. Luongo G. Giabattoni G. Acocella A. Quintessence Publishing 2018)

43. Simon $\mathrm{H}$. Use of transitional implants to support a surgical guide: enhancing the accuracy of implant placement. J Prosthet Dent. 2002 Feb;87(2):229-32.

44. Schneider D, Marquardt P, Zwahlen M, Jung RE. A systematic review on the accuracy and the clinical outcome of computer-guided template-based im- plant dentistry. Clin Oral Implants Res 2009;20(suppl 4):73-86)

45. Daniel Amaral Alves Marlière "Accuracy of computer-guided surgery for dental implant placement in fully edentulous patients: A systematic review” Eur J Dent. 2018 Jan-Mar; 12(1): 153-160.

46. Pettersson A, Komiyama A, Hultin M, Näsström K, Klinge B Clin Implant Dent Relat Res. 2012 Aug; 14(4):527-37.

47. Stübinger S, Buitrago-Tellez C, Cantelmi G. Deviations between placed and planned implant positions: an accuracy pilot study of skeletally supported stereolithographic surgical templates. Clin Implant Dent Relat Res. 2014 Aug; 16(4):540-51.

48. Cassetta M, Giansanti M, Di Mambro A, Stefanelli LV. Accuracy of positioning of implants inserted using a mucosa-supported stereolithographic surgical guide in the edentulous maxilla and mandible. Int J Oral Maxillofac Implants. 2014 Sep-Oct; 29(5):1071-8

49. Derksen W, Wismeijer D, Flügge T, Hassan B, Tahmaseb A. The accuracy of computerguided implant surgery with tooth-supported, digitally designed drill guides based on CBCT and intraoral scanning. A prospective cohort study.Clin Oral Implants Res. 2019 Jul 22.

50. Alevizakos V, Mitov G, Stoetzer M, von See C. A retrospective study of the accuracy of templateguided versus freehand implant placement: A nonradiologic method. Oral Surg Oral Med Oral Pathol 
Oral Radiol. 2019 Sep;128(3):220-226.

51. Tallarico M, Xhanari E, Kim YJ, Cocchi F, Martinolli M, Alushi A, Baldoni EE, Meloni SM. Accuracy of computer-assisted template-based implant placement using conventional impression and scan model or intraoral digital impression: A randomised controlled trial with 1 year of follow-up. Int $\mathrm{J}$ Oral Implantol (New Malden). 2019;12(2):197-206.

52. Skjerven H, Olsen-Bergem H, Rønold HJ, Riis UH, Ellingsen JE. Comparison of postoperative intraoral scan versus cone beam computerised tomography to measure accuracy of guided implant placement-A prospective clinical study. Clin Oral Implants Res. 2019 Jun;30(6):531-541

\section{Figures}
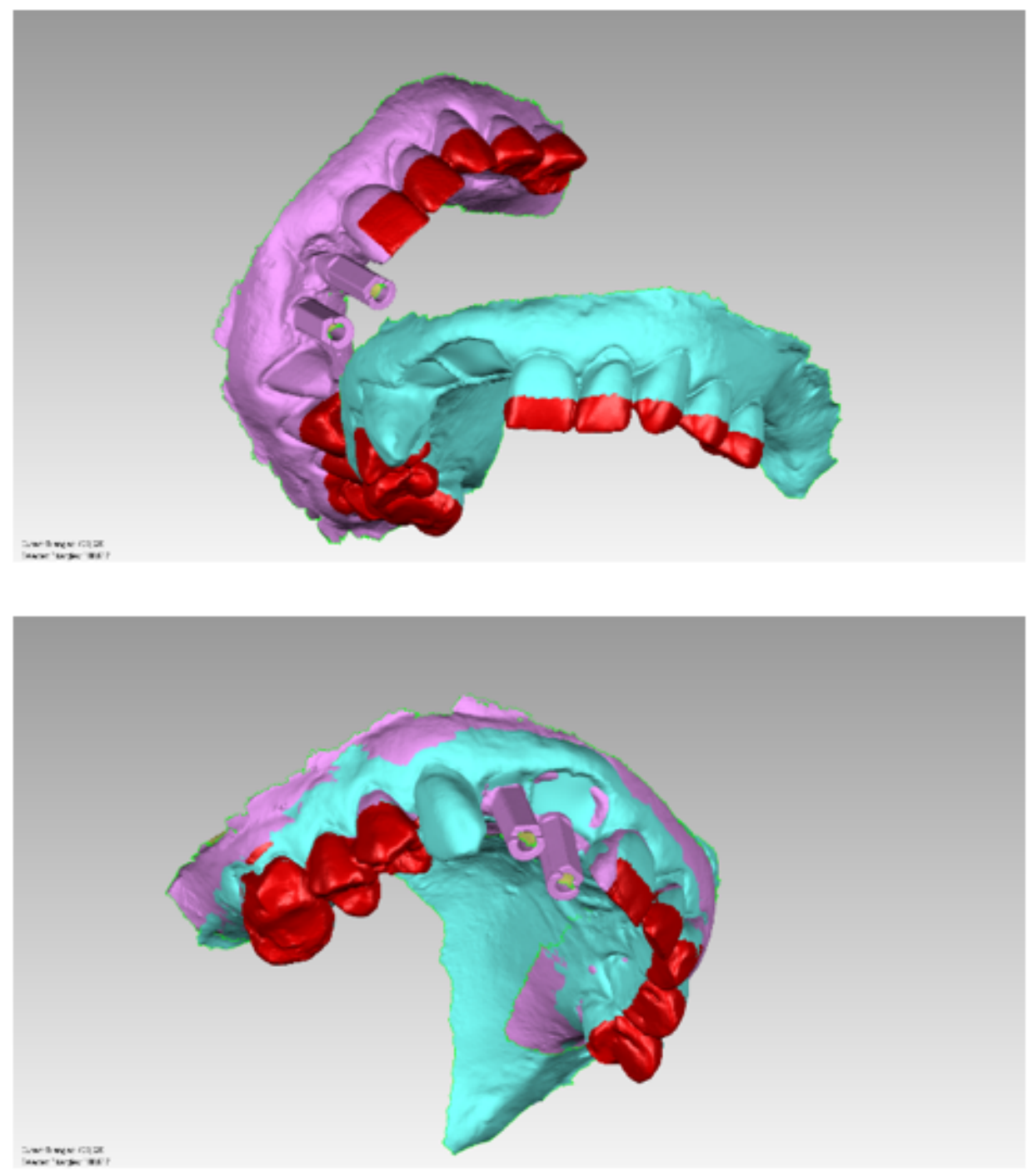

\section{Figure 1}


[Left side picture: before surgery (cyan surface) and after surgery (pink surface) not aligned STL files. The right side picture shows aligned STL files. The red areas were used for superimposition].
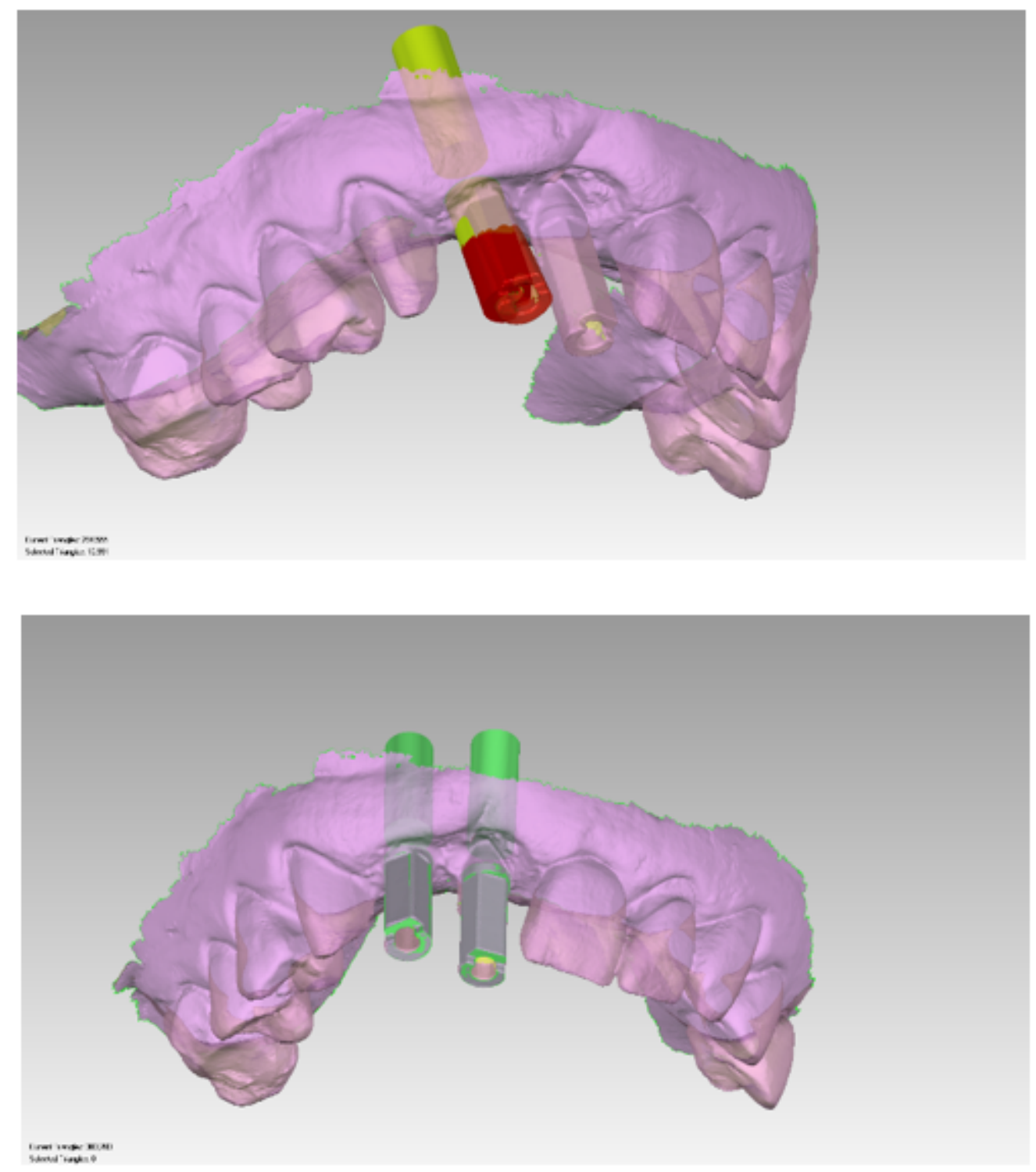

\section{Figure 2}

[The left side picture shows the after-surgery anatomy (pink file with scan abutment) and the planned implant position (yellow file and its scan abutment in the correct position with respect to the platform) from ReaIGUIDE 5.0 library. Geomagic superimposed the red areas, chosen by the user. In the right side picture, all the implants were moved in the after surgery position]. 


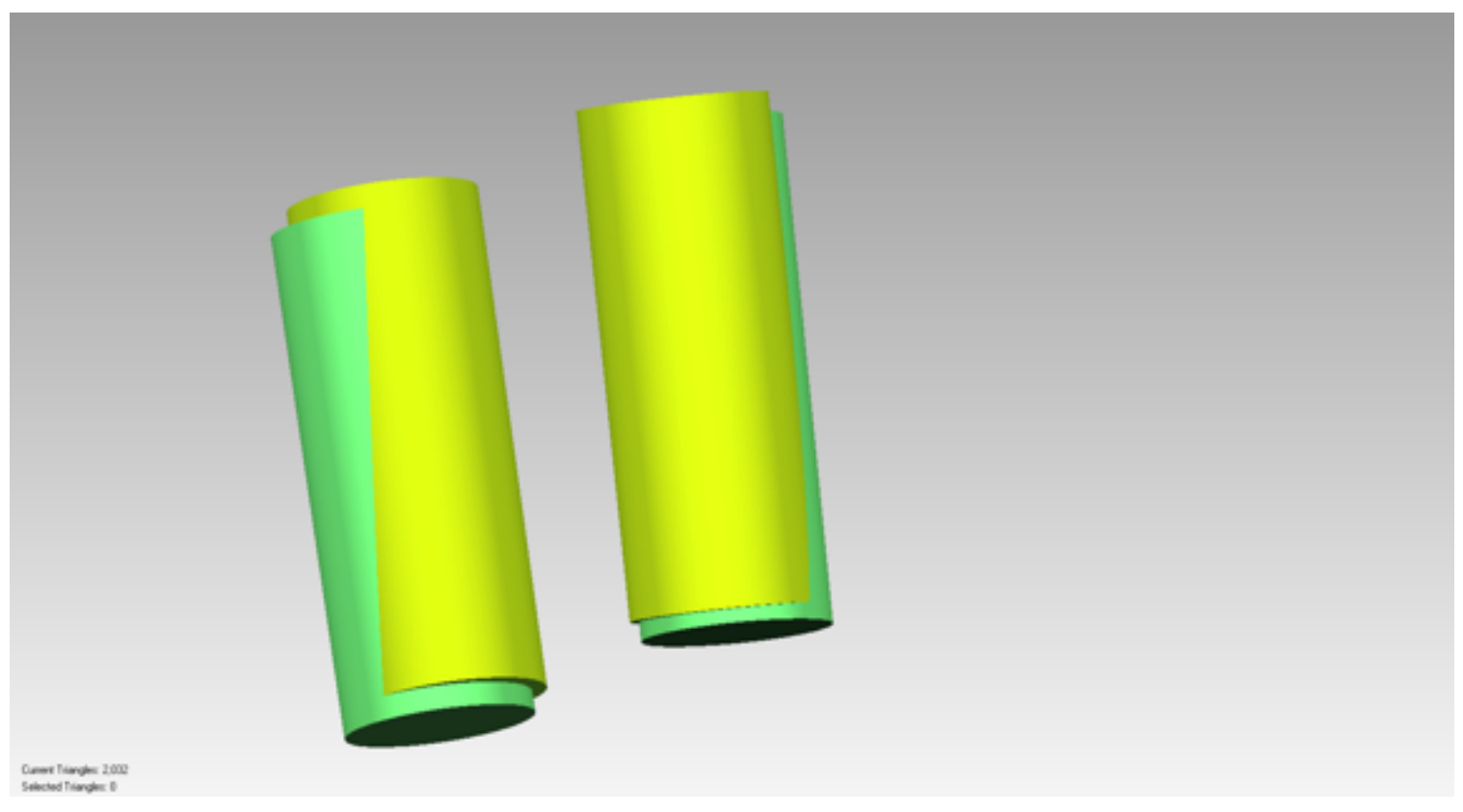

\section{Figure 3}

We simplified before surgery implants (yellow) and after surgery implants (green) into cylinders.

\section{Supplementary Files}

This is a list of supplementary files associated with this preprint. Click to download.

- Formulas.pdf 